\title{
Massa corporal, imagem corporal e desejo por tratamentos estéticos nas diferentes fases do ciclo menstrual
}

\author{
Giulia Silva Araújo ${ }^{1}$, Aline Fernanda Perez Machado¹, Pascale Mutti Tacani², Rogério Eduardo Tacani², \\ Paula Juliana Ferreira Albero ${ }^{3}$, Richard Eloin Liebano ${ }^{4}$
}

\begin{abstract}
RESUMO
Objetivo: analisar a massa corporal, a imagem corporal e o desejo por tratamentos estéticos durante as fases folicular e lútea do ciclo menstrual. Metodologia: este é um ensaio clínico longitudinal prospectivo, no qual 40 mulheres foram acompanhadas durante um ciclo menstrual completo e avaliadas em dois momentos: Fase Folicular e a Fase Lútea; por meio do Body Shape Questionnaire (BSQ) e da Escala de Figuras de Silhuetas, e questionadas quanto ao desejo por tratamentos estéticos quantificado pela Escala Visual Numérica (EVN), durante os meses de janeiro à maio de 2012, na Clínica de Fisioterapia da Universidade Cidade de São Paulo. Resultados: observou-se aumento da massa corporal $(p<0,001)$ e da Escala de Figuras de Silhuetas da FF para FL $(p=0,058)$. Conclusões: houve aumento da massa corporal e alteração da imagem corporal; entretanto, não houve mudança da imagem corporal e do desejo por tratamentos estéticos nas diferentes fases do ciclo menstrual.
\end{abstract}

Descritores: Imagem Corporal; Ciclo Menstrual; Estética; Saúde da Mulher.

\section{Body mass, body image and desire for aesthetic treatments in the different phases of the menstrual cycle}

\begin{abstract}
Objective: analyze the body weight, body image and the desire for aesthetic treatments during the follicular and luteal phases of the menstrual cycle. Methods: this is a prospective longitudinal clinical trial, which forty women were followed for a complete menstrual cycle and evaluated by two times: Follicular Phase and Luteal Phase, for middle of the Body Shape Questionnaire (BSQ) and the Scale Figures Silhouettes and questioned about the desire for aesthetic treatments quantified by Visual Numeric Scale (VNS), during the months of january to may 2012, in the Physical Therapy of São Paulo City University. Results: there was an increase in body mass $(p<0.001)$ and the Scale Figures Silhouettes FF for FL ( $p=0.058)$. Conclusions: increased body mass and impaired body image, however, there was no change in body image and the desire for aesthetic treatments in the different phases of the menstrual cycle.
\end{abstract}

Descriptors: Body Image; Menstrual Cycle; Esthetics; Women's Health.

\footnotetext{
${ }^{1}$ Fisioterapeuta especialista em Fisioterapia Dermatofuncional pela Universidade Cidade de São Paulo (UNICID), Tatuapé, SP, Brasil.

${ }^{2}$ Fisioterapeuta pela Universidade Cidade de São Paulo (UNICID), Tatuapé, SP, Brasil.

${ }^{3}$ Doutora em Fisiologia Humana pelo Instituto de Ciências Biomédicas (ICB), Butantã, SP, Brasil.

${ }^{4}$ Mestre em Cirurgia Plástica Reparadora pela Universidade Federal de São Paulo (UNIFESP), São Paulo, SP, Brasil.
} 


\section{Introdução}

O período reprodutivo feminino normal caracteriza-se por alterações rítmicas mensais que consistem na produção e na secreção dos hormônios femininos e por mudanças correspondentes nos próprios órgãos sexuais. Esse padrão é denominado ciclo sexual feminino ou ciclo menstrual ${ }^{1-3}$. 0 ciclo menstrual normal varia de 21 a 35 dias, com média de 28 dias, podendo ser dividido em três fases distintas: folicular, ovulatória e lútea ${ }^{4-6}$.

A fase folicular é caracterizada por baixos níveis de estradiol e progesterona, que fazem com que o revestimento uterino degenere e se desprenda na menstruação, marcando o primeiro dia do ciclo menstrual. 0 aumento nos níveis dos hormônios luteinizante (LH) e folículo-estimulante (FSH) assinalam o início da fase ovulatória, na qual o nível de estradiol atinge seu máximo e a progesterona se eleva ${ }^{7}$. Na fase lútea, o LH e FSH diminuem, o folículo roto se fecha após ter liberado o óvulo e forma o corpo lúteo, o qual secreta progesterona; caso o óvulo não seja fertilizado, o corpo lúteo se degenera e deixa de produzir progesterona, o nível de estradiol diminui e inicia um novo ciclo menstrual ${ }^{8,9}$. Durante 0 ciclo menstrual, ocorrem oscilações hormonais, principalmente de estrogênio e progesterona, que influenciam a fisiologia feminina. Os hormônios femininos sexuais endógenos e exógenos alteram parâmetros cardiovasculares, respiratórios e metabólicos. Quando se elevam os níveis de progesterona ocorre aumento da temperatura corporal, hiperventilação e elevação do volume plasmático $0^{10,11}$.

Os índices de edema pré-menstrual relatados na literatura chegam a $92 \%$, sendo atribuídos à retenção hídrica, por ação da progesterona, provocando flacidez da parede venosa, com prejuízoà drenageme também por atuarcomo agonista daaldosterona, induzindo a natriurese, imediatamente compensada por aumento de renina ou conversão para desoxicorticosterona ${ }^{12}$.

As frequentes alterações no metabolismo hídrico se manifestam também por dores abdominais, mastalgia e ganho de massa corporal, as quais representam um dos principais responsáveis por influenciar a imagem corporal da mulher. Um dos componentes subjetivos da imagem corporal se refere à satisfação de uma pessoa com seu tamanho corporal ou partes específicas de seu corpo ${ }^{13}$. Assim, considera-se como sendo uma unidade adquirida e dinâmica, portanto alterações corporais como edemas provocam mudanças na imagem corporal ${ }^{14}$.

Atualmente, em função dos padrões de beleza impostos pela sociedade, há uma supervalorização da imagem, onde 0 importante é ter medidas compatíveis com a magreza ${ }^{15}$. Assim, a mulher que tem de lidar com as mudanças frequentes em seu corpo decorrente das alterações hormonais durante 0 ciclo menstrual ${ }^{16}$. 0 excesso de preocupação com a aparência e 0 aumento da insatisfação com o corpo, principalmente com a massa corporal, na contemporaneidade, tem sido o enfoque de muitos estudos científicos, pois a maioria das mulheres acaba recorrendo à tratamentos estéticos, colocando em dúvida a sua real necessidade. Portanto, julga-se necessário realizar um estudo para determinar se tais alterações, constantemente vivenciadas ao longo da menacme, podem aumentar ou não interferir a procura por tratamentos estéticos, motivadas por queixas reais ou fruto de perturbações patológicas advindas do ciclo menstrual. Assim, o objetivo deste estudo foi analisar a massa corporal, a imagem corporal e o desejo por tratamentos estéticos durante as fases folicular e lútea do ciclo menstrual.

\section{Metodologia}

Este é um ensaio clínico longitudinal prospectivo aberto. Foram selecionadas 40 mulheres, sem diagnóstico de doença prévia, com faixa etária entre 18 e 45 anos, com ciclo menstrual regular e que nunca tivessem sido submetidas a qualquer tratamento estético. As voluntárias foram acompanhadas durante um ciclo menstrual completo e avaliadas em dois momentos, sendo um na Fase Folicular (FF), o qual corresponde ao período que compreende entre $013^{\circ}$ e $016^{\circ}$ dias, a partir do primeiro dia do ciclo menstrual; e outro na Fase Lútea (FL), o qual ocorre de 3 a 6 dias antes da menstruação.

O estudo teve início após aprovação do Comitê de Ética em Pesquisa da Universidade Cidade de São Paulo, sob o protocolo de número 13602475. A coleta de dados ocorreu durante os meses de janeiro a maio de 2012, na Clínica de Fisioterapia da Universidade Cidade de São Paulo. As voluntárias assinaram e concordaram com os itens especificados no Termo de Consentimento Livre e Esclarecido.

Os critérios de exclusão foram: uso de corticoides e/ou anticoncepcional não hormonal, mulheres no período pósoperatório menor que seis meses e/ou em tratamento farmacológico e/ou clínico para emagrecimento ou estético, gestantes, puérperas e lactantes. 
As avaliações na FF e FL foram compostas por identificação, anamnese e exame físico por meio da aferição de massa e estatura corporal, para a obtenção do Índice de Massa Corporal (IMC), avaliação da imagem corporal e do desejo por tratamentos estéticos. Todas as avaliações foram realizadas por um único avaliador experiente.

A avaliação da imagem corporal foi feita pelo Body Shape Questionnaire (BSQ) e pela Escala de Figuras de Silhuetas. O BSQ é um questionário autoaplicativo que avalia as preocupações com a forma do corpo, autodepreciação pela aparência física e a sensação de estar "gorda" por meio de 34 perguntas, sendo que cada questão apresenta seis possibilidades de respostas: 1) Nunca, 2) Raramente, 3) Às vezes, 4) Frequentemente, 5) Muito frequentemente e 6) Sempre. Este reflete níveis crescentes de preocupação com a imagem corporal que correspondem às seguintes faixas, de acordo com a somatória de pontos do questionário: até 80 é considerada sem insatisfação; entre 80 e 110, insatisfação leve; entre 111 e 140, insatisfação moderada; e maior que 140 insatisfação grave ${ }^{17,18}$.

A Escala de Figuras de Silhuetas ${ }^{15}$ consiste em um conjunto de nove silhuetas de cada gênero, apresentadas em cartões individuais (numerados de 1 a 9), com variações progressivas na escala de medida, da figura mais magra a mais larga, com IMC médio variando entre 17,5 e $37,5 \mathrm{~kg} / \mathrm{m}^{2}$. A aplicação consistiu em solicitar à voluntária que escolhesse um cartão, dentre os dispostos em série ordenada ascendente, que melhor representasse a silhueta de seu próprio corpo no momento da avaliação. A seguir deveria indicar o cartão com a silhueta que gostaria de ter. Na primeira avaliação, as voluntárias foram questionadas sobre como se classificavam no momento e como gostariam de ser com a Escala em ordem cronológica; as mesmas perguntas foram feitas na segunda avaliação, porém com os cartões das silhuetas organizados de maneira aleatória.

E, por fim, as voluntárias responderam a seguinte pergunta: "Você sente desejo por tratamentos estéticos?", para a qual deveriam assinalar "sim" ou "não". Se a resposta fosse afirmativa, a voluntária deveria quantificar este desejo em uma escala de 0 (zero) a 10 (dez), adaptada da Escala Visual Numérica (EVN), na qual o zero representa nenhum desejo e dez um grande desejo por tratamentos estéticos.

Para análise estatística, utilizou-se o SPSS V16, Minitab 15. A normalidade dos dados foi verificada com o teste de Kolmogorov-Smirnov. A estatística descritiva constou do cálculo de média e desvio padrão (DP). Foi utilizado o Teste t de Student, para variáveis dependentes para avaliar a diferença entre as fases do ciclo menstrual. Para calcular a distribuição da frequência relativa (percentuais) das variáveis qualitativas, foi utilizado o teste de Igualdade de Duas Proporções. $O$ nível de significância foi estabelecido em $p<0,05$.

\section{Resultados e discussões}

Esta pesquisa foi composta por 40 voluntárias com idade média de 26,7 anos $( \pm 6,1)$; quanto à raça $85 \%(n=34)$ eram brancas; em relação aos hábitos de vida, $10 \%(n=4)$ eram fumantes e $60 \%(n=24)$ tinham 0 ciclo menstrual com duração média de 27,5 dias $( \pm 2,7)$.

Quanto ao estado civil, $50 \%(n=9)$ eram casadas, 33,3\% ( $n=6)$ solteiras, $11,1 \%(n=2)$ divorciadas e 5,6\% ( $n=1)$ viúvas; em relação ao nível de escolaridade, 44,4\% $(n=8)$ tinham completado o ensino superior, outras $44,4 \%$ ( $n=8) 0$ ensino médio e $11,1 \%(n=2)$ o ensino fundamental.

No que se refere à massa corporal, observou-se aumento estatisticamente significante da FF para a FL $(p<0,001)$. Consequentemente, houve também aumento estatisticamente significante do IMC ( $p<0,001)$, sendo que, na FF, a média foi de $24,70 \mathrm{Kg} / \mathrm{m}^{2}( \pm 4,8)$ e, na $\mathrm{FL}$, de $24,85 \mathrm{Kg} / \mathrm{m}^{2}( \pm 4,8)$. Não foram demonstradas alterações estatisticamente significantes da imagem corporal por meio do $B S Q(p=0,118)$, sendo que na $F F$, verificou-se pontuação média de 87,7 $( \pm 30,7)$ e na FL, de 85,3 ( $\pm 32,2)$, ambas classificadas como "insatisfação leve". Sobre a Escala de Figuras de Silhuetas, a média da silhueta real na FF foi de 5,03 e na FL a média foi de 5,15, verificou-se um aumento entre as fases com tendência à significância $(p=0,058)$. Quando questionadas sobre o desejo por tratamentos estéticos, $97,5 \%(n=39)$ responderam "sim" em ambas as fases. Destas, na FF, quantificaram este desejo com média de 7,4 ( $\pm 2,2)$; já na FL, a média foi de 7,3 $( \pm 2,3)$, não denotando alterações estatisticamente significantes $(p=0,391)$ (Tabela 1). 
Tabela 1 - Massa corporal, IMC, BSQ, Silhueta real e desejada nas diferentes fases do ciclo menstrual

\begin{tabular}{c|c|c|c|c}
\hline Dados & Fase do ciclo menstrual & Média & $\mathbf{\pm D P}$ & Valor de $\mathbf{p}$ \\
\hline Massa Corporal $(\mathrm{kg})$ & $\mathrm{FF}$ & 65,70 & 12,3 & $<0,001^{*}$ \\
& $\mathrm{FL}$ & 66,10 & 12,3 & \\
\hline $\mathrm{IMC}\left(\mathrm{kg} / \mathrm{m}^{2}\right)$ & $\mathrm{FF}$ & 24,70 & 4,8 & $<0,001^{*}$ \\
& $\mathrm{FL}$ & 24,85 & 4,8 & \\
\hline BSQ & $\mathrm{FF}$ & 87,7 & 30,7 & 0,118 \\
& $\mathrm{FL}$ & 85,3 & 32,2 & \\
\hline Silhueta real & $\mathrm{FF}$ & 5,03 & 1,98 & 0,058 \\
& $\mathrm{FL}$ & 5,15 & 1,90 & \\
\hline Silhueta desejada & $\mathrm{FF}$ & 3,28 & 1,47 & 0,486 \\
& $\mathrm{FL}$ & 3,23 & 1,35 & \\
& & & & \\
\hline
\end{tabular}

Legenda: DP= Desvio padrão; IMC= Índice de massa corporal; BSQ= Body Shape Questionnaire; FL= Fase Lútea; FF= Fase Folicular; *= significância estatística

Observou-se que, quando questionadas em relação à silhueta real, na FF 20\% ( $n=8$ ) responderam que corresponderia a silhueta 4; já na FL 22\% ( $n=9)$ escolheram como silhueta real a 5 . Em relação à silhueta desejada, na FF a média foi de 3,28 e na FL foi de 3,23; não havendo alterações estatisticamente significantes $(p=0,486)$.

\section{Discussão}

Em função do atual padrão de beleza, muitas mulheres recorrem a tratamentos estéticos com possível intenção de reconstruir a própria imagem, a qual pode ser influenciada pela fase do ciclo menstrual. Portanto, o presente estudo teve por objetivo analisar a massa corporal, a imagem corporal e o desejo por tratamentos estéticos durante as fases folicular e lútea do ciclo menstrual.

Sobre à massa corporal e IMC, observou-se aumento significante da FF para a FL, sendo que tal achado corrobora com a literatura, a qual demonstra influência do ciclo menstrual no comportamento alimentar e no desenvolvimento da retenção hídrica na $\mathrm{FL}^{19,20}$. Há uma relação entre 0 ciclo menstrual e as alterações no comportamento alimentar ${ }^{19}$. Sugere-se que, após a ingestão de alimentos ricos em carboidratos, provenientes de alimentos açucarados e doces, a mulher apresenta alívio de sintomas, como depressão, tensão, confusão, tristeza e fadiga, sentindo-se mais calma e alerta. Parece que esse aumento no consumo de carboidratos relaciona-se a uma tentativa de elevar os níveis de serotonina de modo a diminuir os efeitos negativos no humor ${ }^{21,12}$. Portanto, pode ocorrer uma flutuação na massa corporal das mulheres durante a menacme como consequência do aumento da ingestão energética na FL, devido ao aumento do apetite, provocada pela oscilação hormonal ${ }^{19}$.

Já a retenção hídrica ocorre pela alteração do nível de progesterona na fase folicular que pode levar à retenção de líquidos e de sódio, ocasionando aumento do volume plasmático, logo após a ovulação, ${ }^{11,22}$ podendo afetar de $70 \%$ a $92 \%$ das mulheres. ${ }^{23,24}$ Assim, o aumento significante da massa corporal e do IMC durante o ciclo menstrual, pode ser justificado pela retenção hídrica ${ }^{23,24}$ e pelas mudanças relacionadas ao comportamento alimentar ${ }^{25,26}$, porém há necessidade de estudos científicos com maior aprofundamento a cerca do assunto.

Não foram observadas alterações significantes da imagem corporal em relação a FF e a FL, por meio do BSQ. O escore médio do BSQ foi classificado como insatisfação leve em ambas avaliações; entretanto, em estudo anterior ${ }^{27}$ com o delineamento semelhante a este, foi demonstrada grande preocupação das mulheres latinoamericanas em relação ao seu próprio corpo.

Por outro lado, a insatisfação leve com o corpo correlaciona-se com a resposta positiva quanto ao desejo por realizar tratamentos estéticos em ambas as fases do ciclo menstrual, sugerindo que a maioria das mulheres deseja submeter-se a algum tipo de tratamento independente da fase do ciclo menstrual, em função da insatisfação detectada pelo BSQ. Sugere-se que tal preocupação esteja influenciada pelo padrão de beleza imposto atualmente, no qual há uma busca incessante pelas formas corporais cada vez mais magras ${ }^{28}$. 
Observou-se aumento com tendência à significância da Escala de Figuras de Silhueta que correspondeu à situação real da voluntária da FF para FL, sugere-se que tal achado pode estar relacionado com a retenção hídrica já descrita anteriormente $^{13}$. Quanto à escolha das figuras, verificou-se que a silhueta real mais escolhida na FF foi a 4, já a mais escolhida na FL foi a 5, denotando correlação com o aumento da massa corporal, porém sem significância estatística. Já para a silhueta desejada, houve uma variação quanto à escolha das figuras, porém todas as mulheres demonstraram pretensão em estar com imagem corporal abaixo da figura de número 6 , sugerindo que as figuras acima deste número não estariam entre as silhuetas mais desejadas entre as mulheres, por apresentarem formatos corporais mais volumosos.

É importante ressaltar que mulheres que procuram procedimentos estéticos são portadoras de uma expectativa que acarreta em níveis diferentes de ansiedade, justificada pela coragem de investir em seu corpo, pela procura da solução para o seu problema, desejando que o resultado do tratamento proposto seja o mais próximo possível do idealizado, e pela repercussão que sua nova imagem corporal causará para si e para os outros ${ }^{28}$.

Em estudo ${ }^{29}$ que aplicou o teste da silhueta em 128 mulheres universitárias, observou-se que 78,8\% estavam insatisfeitas com a própria imagem corporal. Além disso, 49,2\% apresentavam desejo de reduzir o tamanho da silhueta, enquanto apenas $26,6 \%$ desejavam aumentar. Outro estudo ${ }^{30}$ corrobora com os achados deste, pois o tamanho e forma corporal ideal foram associados às figuras representativas de baixo peso. Os dados apontaram para a relevância das diferenças entre adequação da percepção real e ideal, sinalizando a direção das dificuldades relativas à autopercepção corporal.

A principal relevância deste estudo foi propor o embasamento científico do que ocorre no corpo feminino durante 0 ciclo menstrual para nortear a abordagem e 0 atendimento às mulheres durante 0 ciclo menstrual, observando se há diferença da imagem corporal e desejo por diversos tipos de tratamentos estéticos realizados pela fisioterapia.

Baseando-se nos achados deste estudo, o aumento da massa corporal em função da retenção hídrica, a qual gera edema, é um grande foco de atuação da fisioterapia, pois esta propicia benefícios para pacientes que apresentam diversos tipos de edemas e linfedemas, utilizando frequentemente a técnica de drenagem linfática manual (DLM) ${ }^{31}$. Porém, são escassos ensaios clínicos que investiguem a ação das técnicas de fisioterapia, e principalmente da DLM no edema pré-menstrual ${ }^{32}$. Para muitas mulheres, diminuir o edema pré-menstrual pode ser uma forma de melhorar a aparência física e diminuir o descontentamento com o corpo ${ }^{33,34}$. Neste sentido, a busca por tratamentos para redução da massa corporal, além de ser uma necessidade de saúde física, envolve também necessidades psíquicas e sociais ${ }^{28}$.

Na literatura em geral, observa-se a tentativa de analisar as diferentes respostas fisiológicas, físicas e comportamentais ao longo das fases menstruais. Contudo, as pesquisas existentes possuem pouca consistência na apresentação dos resultados e extensa divergência e contradições entre as metodologias aplicadas. Observa-se que alguns estudos sugerem que pessoas com excesso de peso apresentam maior insatisfação com a imagem corporal, outros demonstram que mesmo naquelas com massa corporal adequada, a prevalência de insatisfação é bastante alta, ${ }^{35}$ ou seja, a relação entre as diferentes fases do ciclo menstrual e a insatisfação com a imagem corporal permanece não resolvida.

Assim, o presente estudo possibilitou o entendimento de que independente da fase do ciclo menstrual, as mulheres sentem desejo por tratamentos estéticos, não se tem mudança em relação à imagem corporal. Porém, sabe-se que com a flutuação da massa corporal, sugere-se que a mulher sinta-se mais insatisfeita com o formato de seu corpo.

\section{Considerações Finais}

Concluiu-se que houve aumento da massa corporal e alteração da imagem corporal, de acordo com a Escala de Figuras de Silhuetas, entre as FF e FL. Entretanto, não houve mudança da imagem corporal por meio do BSQ e do desejo por tratamentos estéticos nas diferentes fases do ciclo menstrual.

\section{Referências Bibliográficas}

1. Dasharathy SS, Mumford SL, Pollack AZ, Perkins NJ, Mattison DR, Wactawski-Wende J et al. Menstrual bleeding patterns among regularly menstruating women. Am J Epidemiol. 2012; 175(6): 536-45.

2. Rhudy JL, Bartley EJ. The effect of the menstrual cycle on affective modulation of pain and nociception in healthy women. Pain. 2010; 149: 365-372. 
3. Bartley EJ, Rhudy JL. Endogenous inhibition of the neciceptive flexion reflex (NFR) and pain ratings during the menstrual cycle in healthy women. Ann Behav Med. 2012; 43: 343-351.

4. McPherson ME and Korfine L. Menstruation across time: menarche, menstrual attitudes, experiences, and behaviors. Women's Health Issues. 2004; 14: 193-200.

5. Williams NI, Reed JL, Leidy HJ, Legro RS, De Souza MJ. Estrogen and Progesterone exposure is reduced in response to energy deficiency in women aged 25-40 years. Human Reproduction. 2012; 25(9): 2328-2339.

6. Barbosa MB, Montebelo MIL, Guirro ECO. Determination of sensory perception and motor response thresholds in different phases of the menstrual cycle. Braz J Phys Ther. 2007; 11: 443-449.

7. Bajaj P, Arendt-Nielsen L, Bajaj P, Madsen H. Sensory changes during the ovulatory phase of the menstrual cycle in healthy women. Eur J Pain. 2001; 5: 135-144.

8. Van der Linden M, Buckingham K, Farquhar C, Kremer Jan AM, Metwally M. Luteal phase support for assisted reproduction cycles. Cochrane Database of Systematic Reviews. 2009.

9. Creinin MD, Keverline S, Meyn LA. How regular is regular? An analysis of menstrual cycle regularity. Contraception. 2004; 70: 289-292.

10. Melegario SM, Simão R, Vale RGS, Batista LA, Novaes JS. A influência do ciclo menstrual na flexibilidade em praticantes de ginástica de academia. Rev Bras Med Esporte. 2006; 12(3): 125-128.

11. Frankovit RJ, Lebrum CM. The athletic woman: menstrual cycle, contraception, and performance. Clin Sports Med. 2000; 19(2): 251-271.

12. Carr-Nangle RE, Johnson WG, Bergeron KC, Nangle DW. Body image changes over the menstrual cycle in normal women. IJED. 1994; 16(3): 267-73.

13. Jappe LM, Gardner RM. Body-image perception and dissatisfaction throughout phases of the female menstrual cycle. Percept Mot Skills. 2009; 108(1): 74-80.

14. Longo MR, Haggard P. An implicit body representation underlying human position sense. Pnas. 2010; 107(26): 11727-11732. 15. Kakeshita IS, Almeida SS. Relação entre índice de massa corporal e a percepção da auto-imagem em universitários. Rev. Saúde Públ. 2006; 40(3): 497-504.

16. Nogueira C, Waldige M, Pinto SJI. Prevalência dos Sintomas da Síndrome Pré-Menstrual. Rev. Bras. Ginecol. Obstet. 2000; 22(6): 347-351.

17. Cooper PJ, Taylor MJ, Cooper Z, Fairburn CG. The development and validation of the Body Shape Questionnaire. IJED. 1987; 4: 485-494.

18. Cordas TA, Neves JEP. Escalas de avaliação de transtornos alimentares. Rev Psiquitr Clín. 1999; 26(1): 41-47.

19. Santos LAS, Soares C, Dias ACG, Penna N, Castro AOS, Azeredo VB. Estado nutricional e consumo alimentar de mulheres jovens na fase lútea e folicular do ciclo menstrual. Rev. Nutr. 2011; 24(2): 323-331.

20. Racine SE, Culbert KM, Keel PK, Sisk CL, Burt SA, Klump KL. Diferrential associations between ovarian hormones and disordered eating symptoms across the menstrual cycle in women. Eat Disord. 2012; 45: 333-344.

21. Cross GB, Marley J, Miles $\mathrm{H}$, Willson K. Changes in nutrient intake during the menstrual cycle of overweight women with premenstrual syndrome. Br J Nutr. 2001; 85(4): 475-82.

22. Backtrom T, Andreem L, Birzniece V, Bjorn I, Johansson IM, Nordenstam HM et al. The role of hormones and hormonal treatments in premenstrual syndrome. CNS Drugs. 2003; 17(5): 325-342.

23. Augusto N, Urbanetz AA. Síndrome da tensão pré-menstrual aspectos clínicos e fisiopatologia. Femina. 1985; 13: 682-690.

24. Costa YR, Fagundes RLM, Cardoso BR. Ciclo menstrual e consumo de alimentos. Rev Bras Nutr Clín. 2007; 22(3): 203-209.

25. Thys-Jacobs S, Starkey P, Bernstein D, Tian J. Calcium carbonate and the premenstrual syndrome: effects on premenstrual and menstrual symptoms. Am J Obstet Gynecol. 1998; 179(2): 444-452.

26. Novotny PP. Síndrome pré-menstrual (SPM): como tratar e evitar. 2a Edição. Editora: Ediouro. Rio de Janeiro,1994. p.77-82.

27. Rodriguéz S, Cruz S. Evaluación de La imagen corporal em mujeres latinoamericanas residentes en Guipúzcoa. Anales de Psicologia. 2006; 22(2): 186-199.

28. Auricchio AM, Massarollo MCKB. Procedimentos estéticos: percepção do cliente quanto ao esclarecimento para tomada de decisão. Rev. Esc. Enferm. 2007; 41(1): 13-20.

29. Coqueiro RS, Petroski EL, Pelegrini A, Barbosa AR. Insatisfação com a imagem corporal: avaliação comparativa da associação com estado nutricional em universitários. Rev Psiquiatr. 2008; 30(1): 31-168.

30. Almeida GAN, Santos JE, Pasian SR, Loureiro SR. Percepção de tamanho e forma corporal de mulheres: estudo exploratório. Psicologia Est. 2005; 10(1): 27-35. 
31. Ciucci JL, Krapp JC, Soraccco JE, Ayguavella J, Marcovecchio LD, Salvia C et al. Clínica e evolução na abordagem terapêutica interdisciplinar de 640 pacientes com linfedema durante 20 anos. J Vasc Bras. 2004; 3(1): 72-76.

32. Ferreira JJ, Machado AFP, Tacani R, Saldanha MES, Tacani PM, Liebano RE. Drenagem linfática manual nos sintomas da síndrome pré-menstrual: estudo piloto. Fisioter Pesq. 2010; 17:75-80.

33. Foster GD, Wadden TA, Vogt RA. Body image in obese before, during, and after weight loss treatment. Health Psychology 1997; 16(3): 226-229.

34. Leonhard ML, Barry NJ. Body image and obesity: effects of gender and weight on perceptual measures of body image. Addictive Behaviors. 1998; 23(1): 31-34.

35. Conti MA, Frutuoso MFP, Gambardella AMD. Excesso de peso e insatisfação corporal em adolescentes. Rev Nutr. 2005; 18: 491-499.

\section{Giulia Silva Araújo}

Endereço para correspondência - Rua: Cesário Galeno, n 448/475, CEP: 03071-000, Cidade: Tatuapé, SP, Brasil

E-mail: giuliasa@hotmail.com

Lattes: http://lattes.cnpq.br/4004068247988424

Aline Fernanda Perez Machado - lifpm@yahoo.com.br

Pascale Mutti Tacani - pascale.tacani@hotmail.com

Rogério Eduardo Tacani - rtacani@uol.com.br

Paula Juliana Ferreira Albero - paju02@gmail.com

Richard Eloin Liebano - liebano@gmail.com

Enviado em 28 de janeiro de 2014.

Aceito em 11 de dezembro de 2014. 
